\title{
EVALUACIÓN DEL DESEMPEÑO TÉRMICO DE UN PROTOTIPO CON CHIMENEA SOLAR E INTERCAMBIADOR TIERRA-AIRE
}

\section{EVALUATION OF THE THERMAL PERFORMANCE OF A PROTOTYPE WITH SOLAR CHIMNEY AND GROUND-AIR EXCHANGER}

\author{
MSc. Javier Orozco Ospino *, Ing. Carlos Guerrero Granados* \\ * Fundación Universitaria del Área Andina, Facultad de Ingeniería y ciencias básicas, \\ Grupo de Investigación e Innovación en Ingeniería Civil. Transv 22 Bis \#4-105, \\ Valledupar, Cesar, Colombia. \\ Teléfono (+57) (5) 5832222. \\ E-mail: \{jaorozco, caguerrero\}@ areandina.edu.co
}

Resumen: En esta investigación se evalúa el desempeño térmico de un prototipo de vivienda con chimenea solar e intercambiador de calor tierra-aire. Se construyeron dos prototipos de vivienda a escala, con iguales dimensiones y materiales, expuestos a la radiación solar; uno de ellos dotado con un sistema de ventilación conformado por intercambiador tierra-aire y chimenea solar. Se registraron los valores de temperatura y humedad relativa en los dos modelos. El prototipo dotado con sistema de ventilación registró en promedio una temperatura inferior en $1{ }^{\circ} \mathrm{C}$ respecto al prototipo de referencia; entre las 10 a.m. y las 4 p.m. la reducción de temperatura fue de $1,9^{\circ} \mathrm{C}$. La temperatura en el interior de los dos prototipos fue mayor que la temperatura exterior. La menor temperatura registrada en el prototipo con sistema de ventilación, y la mayor reducción en el rango horario con mayor temperatura demuestran la viabilidad del sistema.

Palabras clave: Ventilación natural, intercambiador tierra-aire, chimenea solar, prototipo.

\begin{abstract}
This research assesses the thermal performance of a prototype housing to scale with solar chimney and ground-air heat exchanger. Two prototypes of housing were built, with the same dimensions and materials, exposed to solar radiation; one of them equipped with a ventilation system consisting of an earth-air heat exchanger and a solar chimney. The values of temperature and relative humidity were recorded in the two models. The prototype equipped with ventilation system registered an average temperature $1{ }^{\circ} \mathrm{C}$ lower with respect to the reference prototype; between $10 \mathrm{a} . \mathrm{m}$. and the 4 p.m. the temperature reduction was $1.9^{\circ} \mathrm{C}$. The temperature inside the two prototypes was higher than the outside temperature. The lower temperature recorded in the prototype with ventilation system, and the greater reduction in the hourly range with higher temperature demonstrate the viability of the system.
\end{abstract}

Keywords: Natural ventilation; earth-air heat exchanger; solar chimney; prototype. 


\section{INTRODUCCIÓN}

La ciudad de Valledupar, localizada en el departamento del Cesar, está ubicada en un valle en la llanura del Caribe en el norte de Colombia. Tiene un clima cálido muy seco, con una temperatura promedio de $28.4{ }^{\circ} \mathrm{C}$ durante todos los días del año, con una variación que aumenta los mediodías y puede registrar una temperatura máxima media que oscila entre los 33 y $36{ }^{\circ} \mathrm{C}$ (Ideam, 2018), convirtiendo a esta ciudad de casi 500.000 habitantes (Dane, 2018) en una de las más calientes del país. Durante la ocurrencia de un patrón climático recurrente como el fenómeno del Niño, las alteraciones de la temperatura del aire son incluso superiores a $0.5{ }^{\circ} \mathrm{C}$, llegando a clasificarse como calentamiento de tipo severo (Ideam, 2018) (JS Molina, JFG Díaz. 2013),

Por otra parte, la humedad relativa del aire varía entre 56 y 74\%, registrando valores mayores en la época lluviosa del segundo semestre del año entre los meses de agosto y noviembre (Ideam, 2018).

Las principales fuentes de calor no deseadas al interior de una vivienda son las ganancias directas solares por las ventanas, la transmisión de calor desde el exterior a través de los materiales de la estructura, y los aportes internos producidos por el equipamiento y los habitantes; estos fenómenos hacen que en las viviendas expuestas a la radiación solar se alcancen mayores temperaturas que en el exterior, por lo que requieren estrategias bioclimáticas de actuaciones directas sobre el control de la radiación solar, tales como cubiertas, aleros, cerramientos, vidrios, fachadas, arborización, etc. y actuaciones indirectas de refrigeración mecánica o pasiva (Morón et al., 2015).

Sin embargo, los proyectos de vivienda de interés social tienden a minimizar los costos constructivos en detrimento del confort higrotérmico, lo cual trae como consecuencia un diseño no adaptado a las condiciones climáticas, y adicionalmente el nivel socioeconómico de sus habitantes no les permite en general acceder a sistemas de refrigeración mecánica que permitan mejorar las condiciones internas de sus viviendas, debido al costo de la energía convencional (Flores et al., 2007)

Los sistemas de refrigeración pasiva se basan en la interacción de la vivienda con su entorno, de tal forma que la ventilación se produce por la acción del viento o la existencia de un gradiente de temperatura entre los puntos de entrada y salida del aire, para mantener la calidad del aire interior y reemplazar el aire viciado por otro más limpio, evitando el exceso de calor soportado por el cuerpo; con lo anterior, se pretende eliminar o reducir el uso de refrigeradores mecánicos y su consumo de energía (Morón et al., 2015) (DC Rojas, OR Alvarado. 2017).

Sin embargo, las soluciones con sistemas de refrigeración pasiva no se pueden generalizar ya que el emplazamiento de la vivienda presenta características propias tales como factores climáticos, biológicos y culturales, que juegan un papel determinante a la hora de plantear respuestas (Pérez et al., 2013). La simulación del flujo de aire en sistemas de ventilación híbridos, compuestos por intercambiador de calor tierra-aire y chimenea solar, está lejos de ser completamente desarrollada, ya que los modelos existentes no son suficientes para evaluar su rendimiento (Zhang y Haghighat, 2005). A la fecha no hay datos que validen el uso de estos sistemas a las condiciones atmosféricas de la ciudad de Valledupar. (YE Santafé, BD Chaparro. 2013).

En esta investigación se evalúa el desempeño térmico de un prototipo a escala con chimenea solar e intercambiador de calor tierra-aire, y se comparan los valores de temperatura y humedad relativa en su interior con los registrados en otro prototipo de iguales dimensiones y materiales, pero sin el sistema de refrigeración. Se realiza un estudio preliminar para evaluar la conveniencia de implementar este sistema de refrigeración pasiva en viviendas de interés social en las condiciones climáticas de la región de Valledupar, lo cual impactará a los usuarios directos al permitirles tener acceso a alternativas de control del ambiente interior, o la disminución de los costos energéticos si tienen la posibilidad de acceder a los mecanismos de climatización artificial.

\section{Materiales y métodos}

Para el desarrollo de esta investigación se construyeron dos prototipos de vivienda a escala con iguales dimensiones y materiales, expuestos a la radiación solar; uno de ellos se dotó con un sistema de ventilación conformado por intercambiador tierra-aire y chimenea solar; el otro prototipo es completamente cerrado, debido a que se pretende evaluar el funcionamiento del sistema de ventilación en el sentido de modificar algunas de las variables climáticas que influyen en el confort en este tipo de ambientes. 
Los modelos a escala tienen dimensiones reducidas, por lo cual los resultados obtenidos se consideran preliminares; en una investigación posterior se debe implementar el sistema de ventilación en un modelo a escala real y con los materiales comúnmente usados en la construcción de viviendas, con el fin de establecer resultados definitivos.

Cada uno de los prototipos tiene $1 \mathrm{~m}$ de ancho, $1 \mathrm{~m}$ de longitud, $1 \mathrm{~m}$ de altura y cubierta a dos aguas con $1,40 \mathrm{~m}$ de altura en la parte central; la estructura se conformó con listones de madera 5x5 $\mathrm{cm}$ de sección transversal, con 4 columnas empotradas en el suelo con una base de concreto. Las paredes y el piso se construyeron en lámina de madera prensada de $1 \mathrm{~cm}$ de espesor; este material se seleccionó por su baja conductividad térmica con el fin de contribuir a aislar el ambiente interior, ya que las dimensiones reducidas de los modelos aumentan considerablemente la influencia de las condiciones ambientales externas. La cubierta se instaló en lámina de acero galvanizado calibre 18 . En la Fig. 1 se muestran estas características.

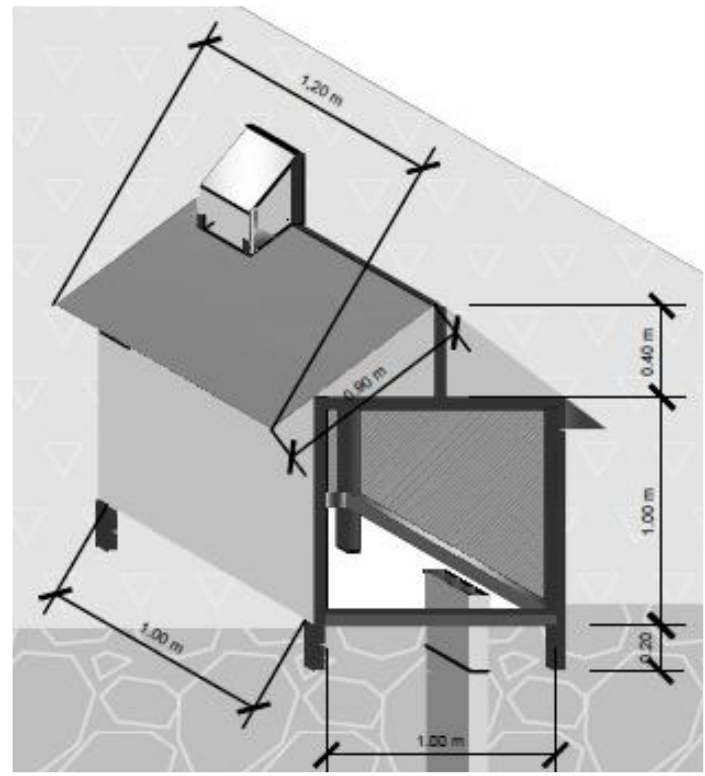

Fig. 1 Prototipo de vivienda. Fuente: autores.

El intercambiador tierra-aire se construyó con bloques de arcilla de $23 \times 8 \mathrm{~cm}$ de sección transversal y $80 \mathrm{~cm}$ de longitud, los cuales disponen de 3 conductos de $5 \mathrm{~cm}$ de diámetro; la longitud total del intercambiador es $10 \mathrm{~m} \mathrm{y}$ se instaló a una profundidad de $1 \mathrm{~m}$, tal como se indica en la Fig. 2 (ver anexo).

Debido a que el material utilizado tiene baja conductividad térmica, se aplica pintura negra en la parte interna para una mayor absorción de la radiación solar, que se transforma en energía térmica y permite que la chimenea se caliente más. La entrada de aire en la parte inferior es de 26x10 cm y la salida está localizada en la parte superior de la pared vertical trasera con dimensiones de $26 \times 5 \mathrm{~cm}$. En la fig. 3 se muestra una vista lateral de la chimenea con los detalles de las aberturas de entrada y salida del aire.

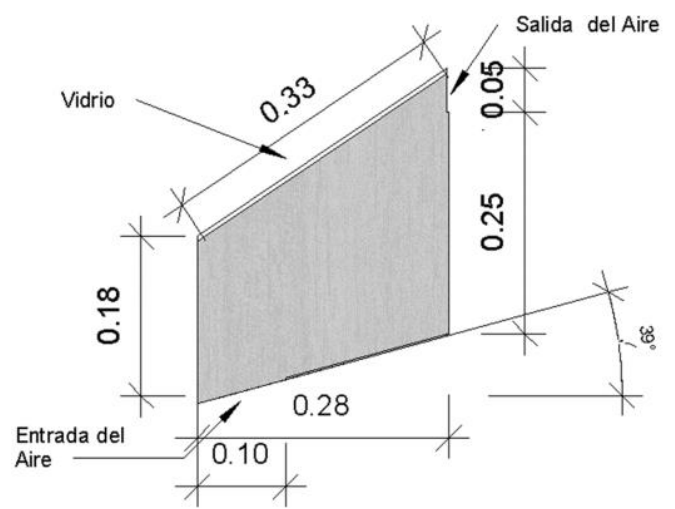

Fig. 3 Vista lateral de la chimenea solar (medidas en metros). Fuente: autores.

Teniendo en cuenta que las principales variables climáticas que influyen sobre el confort son la temperatura del aire, la humedad, el movimiento del aire, y la radiación solar (Fernández, 1994), se seleccionan para el análisis las dos primeras variables, las cuales están influenciadas por las dos últimas en el funcionamiento del sistema de ventilación propuesto.

El registro de los valores de temperatura y humedad relativa en el interior de los dos prototipos se realizó con un termohigrómetro Dataloger RHT20 con las siguientes características: interfaz USB para descarga de datos, frecuencia de muestreo de datos seleccionable desde 1 segundo hasta 24 horas, y software de análisis compatible con Windows 2000/XP/Vista/7. La frecuencia de muestreo de datos se programó con un intervalo de 10 minutos, desde las 6:00 hasta las 20:00, durante un período de 24 días; las mediciones se iniciaron el 12 de marzo de 2016 y finalizaron el 4 de abril. No se registró el período de la noche porque el mayor movimiento de aire generado por una chimenea solar se presenta cuando más se calienta, proporcionando mayor ventilación en el mediodía (Lal et al., 2013). Los datos registrados se descargaron cada seis días y se almacenaron en un computador, inicialmente en archivos individuales.

Considerando que el alcance de la investigación es evaluar la variación de la temperatura y humedad 
relativa generada por el sistema de ventilación en un ambiente cerrado, la información climática en el exterior se registra como referencia desde el portal de internet Freemeteo.com (Freemeteo, 2016), que a su vez obtuvo los datos de la estación meteorológica del Instituto de Hidrología, Meteorología y Estudios Ambientales (IDEAM), localizada en el aeropuerto Alfonso López Michelsen ubicado en la ciudad de Valledupar; los datos de temperatura y humedad están disponibles con intervalos de una hora para el período de estudio.

El procesamiento de los datos y las gráficas se realizó en Microsoft Excel. Para el cálculo de los valores promedio de temperatura y humedad relativa en el interior de los prototipos se usaron todos los datos registrados con intervalo de 10 minutos; en la elaboración de las gráficas se emplearon los datos con intervalo de una hora para que coincidieran con los datos disponibles de las condiciones climáticas en el exterior. También se analizó el día más cálido en relación con los resultados promedios.

\section{Resultados y análisis}

La Fig. 4 muestra los valores promedios de temperatura registrados durante los días de análisis para el ambiente exterior y el interior de los prototipos de vivienda con $y$ sin sistema de ventilación.

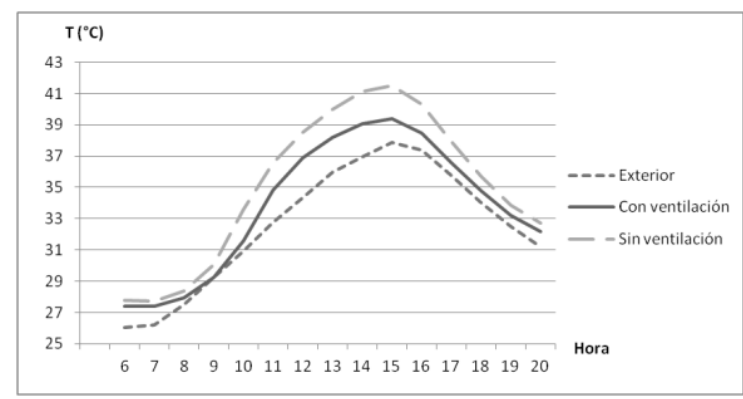

Fig. 4 Temperatura promedio exterior e interior en los prototipos de vivienda. Fuente: autores.

El prototipo dotado con sistema de ventilación registró durante todas las horas del día una temperatura inferior respecto al prototipo de referencia, con un valor promedio de $1{ }^{\circ} \mathrm{C}$. Entre las 10 a.m. y las 4 p.m. la reducción de temperatura fue mayor, con un promedio de $1,9{ }^{\circ} \mathrm{C}$, alcanzando un valor máximo de $2,2{ }^{\circ} \mathrm{C}$ a las 3 p.m. Por otra parte, se obtuvo una reducción promedio de $1,8^{\circ} \mathrm{C}$ en los períodos en los cuales la temperatura del prototipo fue mayor de $30^{\circ} \mathrm{C}$.
En el día más cálido fue mayor la reducción de temperatura en el prototipo con sistema de ventilación respecto al prototipo de referencia, con un valor promedio de $1,5^{\circ} \mathrm{C}$. Entre las 10 a.m. y las 4 p.m. la reducción de temperatura aumentó a $2,4^{\circ} \mathrm{C}$, con valores máximos de $2,8^{\circ} \mathrm{C}$ a las 3 p.m. En la franja horaria con temperatura mayor de 30 ${ }^{\circ} \mathrm{C}$ en el prototipo, la reducción promedio fue igual a la registrada en la totalidad de los días, es decir $1,8{ }^{\circ} \mathrm{C}$.

Los datos anteriores reflejan la capacidad de autobalance de la chimenea solar, considerando las variaciones de temperatura en el transcurso del día y en relación con el día más cálido, ya que entre más caliente es el día, más se calienta la chimenea, y por lo tanto más rápido es el movimiento del aire (Pérez et al., 2013), generando a su vez una mayor reducción de la temperatura en el interior del prototipo en las horas del mediodía, cuando es más necesario en climas cálidos (Lal et al., 2013).

No obstante, la temperatura en el interior de los dos prototipos fue mayor que la temperatura exterior. La diferencia promedio en el prototipo con sistema de ventilación fue $1,2{ }^{\circ} \mathrm{C}$ con un valor máximo de $2,5^{\circ} \mathrm{C}$ a las 12 del día; en el prototipo de referencia la diferencia promedio fue de $2,5{ }^{\circ} \mathrm{C}$, alcanzando un máximo de $4,2{ }^{\circ} \mathrm{C}$, el cual se registró a las 12 amy a las 2 p.m.

En el día más cálido, la diferencia promedio en el prototipo con sistema de ventilación en relación con la temperatura exterior fue de $1,3{ }^{\circ} \mathrm{C}$, con un valor máximo de $2,9{ }^{\circ} \mathrm{C}$ a las 2 p.m.; en el modelo sin sistema de ventilación, esta diferencia fue de $2,8{ }^{\circ} \mathrm{C}$ con un valor máximo de $5,4^{\circ} \mathrm{C}$ a las 2 p.m. La mayor temperatura registrada en el interior de los dos prototipos de vivienda en relación con la del ambiente exterior está determinada en gran medida por la baja altura del techo, lo cual permite que la alta temperatura de la cubierta en acero galvanizado ejerza una influencia determinante sobre las condiciones del clima en el interior.

Este comportamiento afecta el rendimiento del sistema de ventilación, ya que no se genera el gradiente de temperatura necesario entre la chimenea solar y el interior del prototipo, haciendo más lento el movimiento del aire y reduciendo su ingreso a menor temperatura desde el intercambiador tierra-aire.

Por lo anterior, los resultados obtenidos se consideran preliminares ya que para llegar a conclusiones definitivas se requiere implementar el 
sistema de ventilación en un prototipo a escala real y con los materiales utilizados comúnmente en la construcción de viviendas, con el fin de desarrollar un modelo útil que no se afecte en gran medida por las condiciones de frontera.

La Fig. 5 muestra los valores promedios de humedad relativa para el ambiente exterior y el interior de los prototipos de vivienda con y sin sistema de ventilación. El prototipo con sistema de ventilación registró durante todas las horas del día una humedad relativa mayor que la del prototipo de referencia, con una diferencia promedio de $4,8 \%$, alcanzando un valor máximo de $8,9 \%$ alrededor de las 10 a.m.

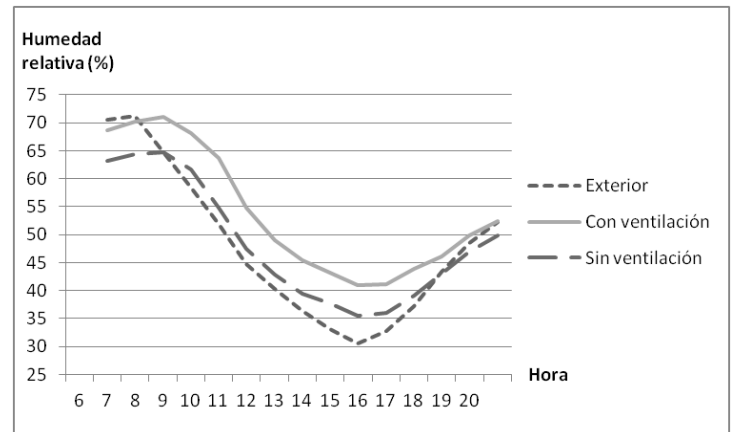

Fig. 5 Humedad relativa exterior e interior en los prototipos de vivienda. Fuente: autores

La mayor humedad relativa registrada en el prototipo con sistema de ventilación respecto al prototipo de referencia está acorde con la relación inversamente proporcional entre la temperatura y la humedad relativa, ya que el modelo con sistema de ventilación registró una temperatura inferior; esto se cumple porque en estas condiciones no existen aportes adicionales significativos de humedad. Por el contrario, en el prototipo de referencia se obtuvo una mayor humedad y temperatura que en el exterior, no cumpliéndose la relación inversa entre estos parámetros.

Por otra parte, en la mayor parte del día la humedad relativa en el interior de los dos modelos fue superior a la del ambiente exterior; en el modelo con ventilación la diferencia promedio fue 7,3\% entre las 8:00 y las 20:00 horas, con un valor máximo de $11,8 \%$ a las 10:00; en el modelo patrón la diferencia promedio entre las 9:00 y las 17:00 horas fue $3,2 \%$ con un máximo de $5,0 \%$ a las 15:00.

\section{CONCLUSIONES}

El prototipo dotado con un sistema de ventilación compuesto por un intercambiador de calor tierra- (Consultado: 16 de junio 2018) aire y una chimenea solar registra una menor temperatura respecto al prototipo de referencia, lo cual demuestra la viabilidad del sistema. Esto se refuerza por el hecho de que la reducción de temperatura es mayor entre las 10 a.m. y las 4 p.m. debido a la mayor radiación que actúa sobre la chimenea, aumentando el gradiente de temperatura respecto al interior del prototipo y por consiguiente el movimiento y renovación del aire interior proveniente del intercambiador.

El rango horario anterior coincide en gran parte con el período de mayor temperatura, por lo que el sistema tiene utilidad práctica; lo anterior se refrenda por la mayor reducción de temperatura que se obtiene para valores superiores a $30{ }^{\circ} \mathrm{C}$ en el interior del prototipo.

La menor reducción de temperatura fuera del rango horario referenciado anteriormente indica que para optimizar el rendimiento del sistema de ventilación durante cualquier hora del día, se requiere ventilación asistida, ya que la falta de radiación solar limita o anula el funcionamiento de la chimenea. Un extractor eólico en la salida de la chimenea puede ser una buena opción para permitir el funcionamiento del sistema en horarios nocturnos.

No obstante, la temperatura en el interior de los modelos, incluyendo el prototipo con sistema de ventilación, fue mayor que la temperatura del ambiente exterior, debido a que sus dimensiones a escala reducida permiten que la variación de las propiedades de los materiales utilizados ejerzan una influencia determinante en el volumen de aire interior; por lo anterior, los resultados obtenidos no se consideran definitivos, ya que para obtenerlos es necesario implementar el sistema de ventilación en un modelo a escala real y con los materiales utilizados comúnmente en la construcción de viviendas.

\section{REFERENCIAS}

IDEAM. (2018). Características climatológicas de ciudades principales y municipios turísticos, Ideam, Bogota.

Departamento Administrativo Nacional de Estadísitica. (2018). Colombia. Proyecciones de población municipales por área, Bogotá. https://www.dane.gov.co/files/investigaciones/pobl acion/proyepobla06_20/ProyeccionMunicipios200 5_2020.xls 
DC Rojas, OR Alvarado. (2017), Ontología para los sistemas holónicos de manufactura basados en la unidad de producción. Revista Colombiana de Tecnologías de Avanzada ISSN: 1692-7257.

JS Molina, JFG Díaz. (2013), Implementación de un sistema tipo Scada para mejorar los procesos de secado y cocción de la ladrillera sigma Ltda. Revista Colombiana de Tecnologías de Avanzada ISSN: 1692-7257.

Morón, C., García, A., Ferrández D. y Hosokawa, K. (2015). Acondicionamiento Pasivo de una Vivienda en la Sierra de Madrid, Anales de Edificación, vol. $1, \mathrm{n}^{\circ} 1$.

Flores, L., Flores L., S y Filippín, C. (2007). Comportamiento térmico de invierno y verano de viviendas de interés social en la provincia de Salta, Avances en Energías Renovables y Medio Ambiente, vol. 11.

Pérez, H., Flores, J. y López, A. (2013). Modelo de ventilación inducida para la vivienda en clima cálido húmedo: sistema chimenea solar, Foro Latinoamericano de Ingeniería, $\mathrm{Foz}$ de Iguazu. https://www.unila.edu.br/sites/default/files/fil es $/ 26 \% 20$ -

$\% 20$ Modelo\%20de\%20ventilaci\%C3\%B3n\% 20inducida\%20para\%20la\%20vivienda\%20e $\mathrm{n} \% 20$ clima\%20c\%C3\%A1lido\%20h\%C3\%B Amedo\%20sistema\%20chimenea\%20solar.pd $\mathrm{f}$

(Consultado: 10 de febrero 2016)

Zhang, J. y Haghighat, F. (2005). Simulation of earth-to-air heat exchangers in hybrid ventilation systems, Ninth International IBPSA Conference, Building Simulation, Montreal, Canada.

Fernández G., F. (1994). Clima y confortabilidad humana: aspectos metodológicos, Serie Geográfica, vol. 4.

Lal, S., Kaushik, S. C. y Bhargava, P. K. (2013). A case study on solar chimney-assisted ventilation for residential building in India, International Journal of Energy Sector Management, vol. 7, $\mathrm{n}^{\circ} 4$.

Freemeteo. (2016). El tiempo, previsiones meteorológicas para todas las ciudades. http://freemeteo.com.co/eltiempo/?language= spanishar\&country $=$ colombia

(Consultado: 20 de junio 2016)

YE Santafé, BD Chaparro..., (2013), Deteccción de patrones característicos con transformadas wavelet en señales electromiográficas del cuádriceps Revista Colombiana de Tecnologías de Avanzada ISSN: 1692-7257

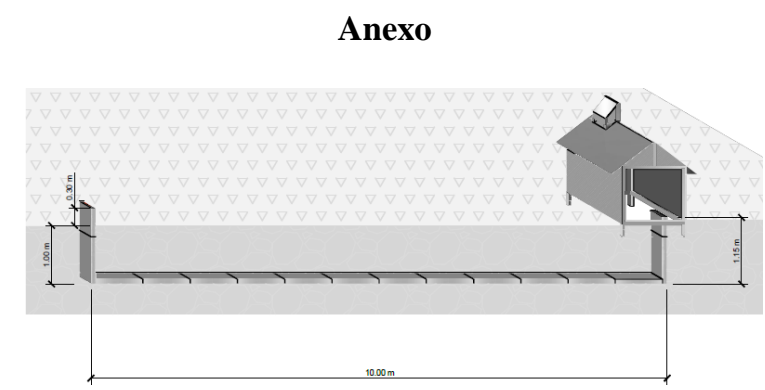

Fig. 2 Sistema de ventilación compuesto por intercambiador tierra-aire y chimenea solar. Fuente: autor 